\title{
Skin metastasis from an occult esophageal adenocarcinoma
}

\author{
F Fereidooni $M D^{1}$, K Kovacs MD PhD², MR Azizi MD ${ }^{1}, M$ Nikoo $M D^{3}$
}

\begin{abstract}
F Fereidooni, K Kovacs, MR Azizi, M Nikoo. Skin metastasis from an occult esophageal adenocarcinoma. Can J Gastroenterol 2005;19(11):673-676.

Metastases to the skin from carcinoma arising in other organs are uncommon, yet they may be the first presentation of neoplastic disease. They usually originate from primary tumours in the breast, lung or colon. Skin metastases from esophageal adenocarcinoma are extremely rare. A unique case of an otherwise healthy patient who presented with a small, painless, mobile, clinically localized facial skin nodule is reported. A biopsy revealed metastatic adenocarcinoma, and subsequent investigations detected the primary tumour in the esophagus, despite no symptoms.
\end{abstract}

\section{Des métastases cutanées émanant d'un adénocarcinome œsophagien occulte}

Key Words: Cutaneous metastases; Esophageal adenocarcinoma; Histology; Oncology; Pathology

\begin{abstract}
Les métastases cutanées provenant de carcinomes d'autres organes ne sont pas courantes, mais elles peuvent pourtant constituer la première manifestation d'une maladie néoplasique. D'ordinaire, elles proviennent de tumeurs primaires du sein, du poumon ou du côlon. Des métastases cutanées émanant d'un adénocarcinome œsophagien occulte sont d'une extrême rareté. Est exposé le cas unique d'un patient autrement en santé qui a consulté en raison d'un petit nodule cutané mobile et indolore cliniquement localisé au visage. Une biopsie a révélé la présence d'un adénocarcinome métastatique, et des examens subséquents ont permis de repérer la tumeur primaire de l'œsophage, malgré l'absence de symptômes.
\end{abstract}

$\mathrm{C}$ utaneous metastases are rare. Metastases to the skin from carcinoma of internal organs are uncommon (1), yet they may be the first sign of neoplastic disease $(2,3)$. Skin involvement is the first sign of the cancer in $0.8 \%$ of these patients, and the underlying cancer is undiagnosed in $60 \%$ of cases $(2,4)$. Skin metastasis indicates dissemination and rapid fatal termination of the tumour (5). Esophageal carcinoma has a very poor prognosis, with a five-year survival rate of $8 \%$ to $12 \%$. It is usually diagnosed at an advanced stage, making cure unlikely (6). Cutaneous metastases of esophageal adenocarcinoma are exceedingly rare (6). We report here a unique case of a 62-year-old woman who presented with a small, solitary, clinically localized skin nodule on her face. She had no other symptoms. A biopsy of the lesion revealed a metastatic adenocarcinoma, and subsequent investigations detected the tumour in the esophagus, despite the absence of local symptoms.

Although carcinoma of the internal organs presents uncommonly with skin metastases, it is necessary that any nonhealing ulcers, persistent indurated erythemas and skin nodules of undetermined causes be biopsied to detect cutaneous metastasis of visceral cancer. It should be noted that the growth pattern of skin metastases may be unpredictable and may not reflect that of the primary tumour (4). Here we report a rare case of cutaneous metastasis before the detection of the primary site. The patient was considered to be at early stages of esophageal carcinoma. We present the histopathological features, and the immunohistochemical profile of both the

primary and the metastatic tumours to assess the usefulness of these markers in deciding therapy. To our knowledge, similar cases have not been reported to date.

\section{CASE PRESENTATION}

A 62-year-old woman presented with a small, nonulcerated, round, well-demarcated, dome-shaped, soft, mobile, nontender, painless, nonpruritic and clinically localized skin nodule of $0.5 \mathrm{~cm}$ diameter and of one month duration, over her left cheek. She told that a month and subsequently a week before her admission, her left cheek was bitten accidentally by her twoyear-old grandson. Although the skin lesion was initially considered to be just a reaction to the bites, or a benign fibroma or a subcutaneous cyst, biopsy detected an adenocarcinoma suspicious for metastasis (Figure 1). On follow-up, the patient looked healthy; she had a good appetite and no major complaints. She was a nonsmoker, did not consume alcohol and was not addicted to drugs. Her body weight was within normal range and there was no history of any medication that may have affected her health. Physical examination was unremarkable. Her blood tests were all within normal limits, except for mild anemia. To detect the primary site of the cancer, radiography and endoscopy were used to evaluate the digestive system. She had no history of nausea, vomiting, increased or decreased sialorrhea, difficulty in swallowing, dyspepsia, reflux or heartburn, epigastric pain or discomfort, hematemesis or changes in bowel habits. A barium swallow demonstrated a slight mucosal irregularity in the distal

${ }^{1}$ Pathology Department, Cancer Institute, Tehran University of Medical Sciences, and Shahriar Hospital, Pathology Laboratory, Tehran, Iran;

Divisions of ${ }^{2}$ Pathology and ${ }^{3}$ Cardiology, Department of Laboratory Medicine and Pathology, St Michael's Hospital, University of Toronto,

Toronto, Ontario

Correspondence and reprints: Dr Forouzandeh Fereidooni, 175 Cedar Avenue, Richmond Hill, Ontario L4C 9V3. Telephone 905-883-1499, fax905-780-9577, e-mail ffereidooni@fairsys.com

Received for publication April 19, 2005. Accepted May 19, 2005 


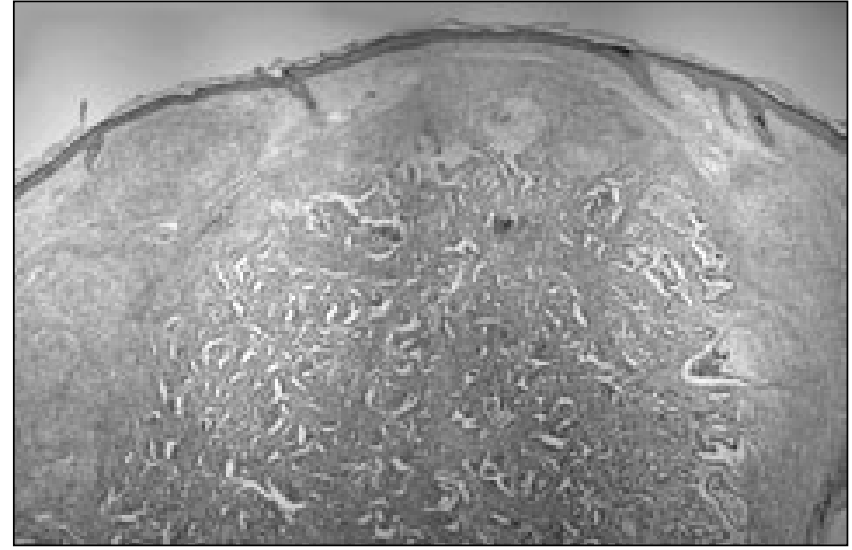

Figure 1) Whole-mount view of skin with metastatic adenocarcinoma. Hematoxylin and eosin staining, original magnification $\times 40$

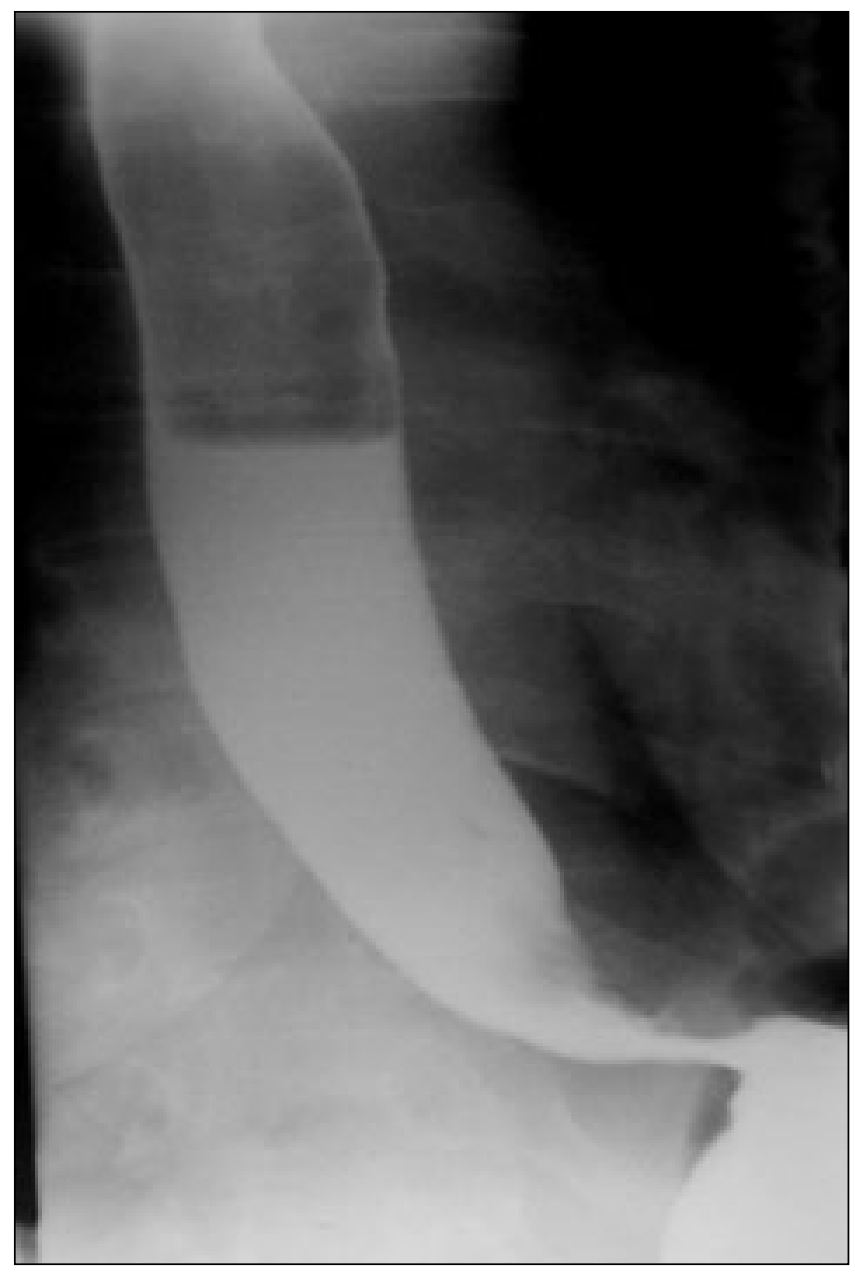

Figure 2) A barium swallow showing deformity of distal esophageal mucosa

esophagus extending to the esophagogastric junction, suggestive of a gastric or esophageal primary tumour (Figure 2). Axial spiral computed tomography scan of the abdomen with contrast revealed thickening in the fundus of the stomach with extension to the cardia, with no peripheral lymphadenopathy. A biopsy of the distal esophagus revealed the characteristic histological features of adenocarcinoma (Figure 3), and an esophageal extension of a gastric carcinoma remained to be excluded. Additional

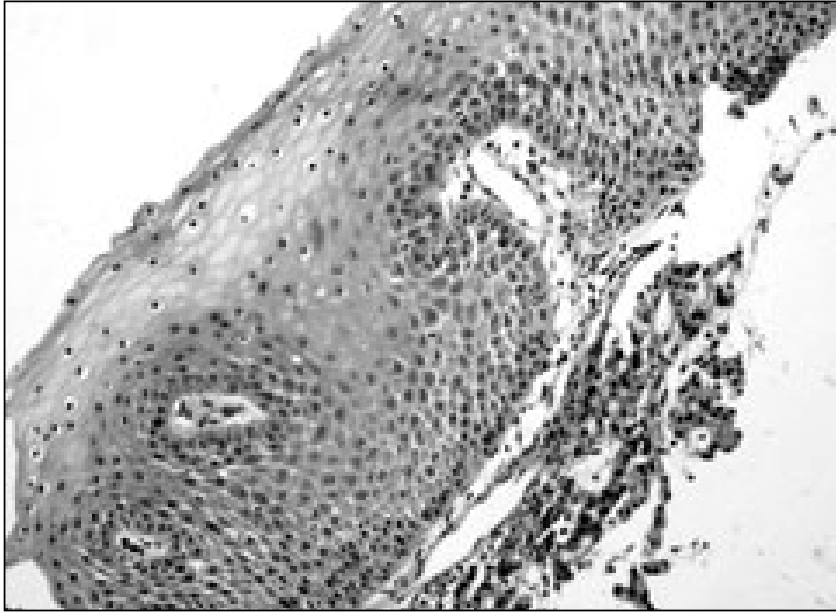

Figure 3) Adenocarcinoma in the esophageal mucosa identical to that in the dermal infiltration. Hematoxylin and eosin staining, original magnification $\times 200$

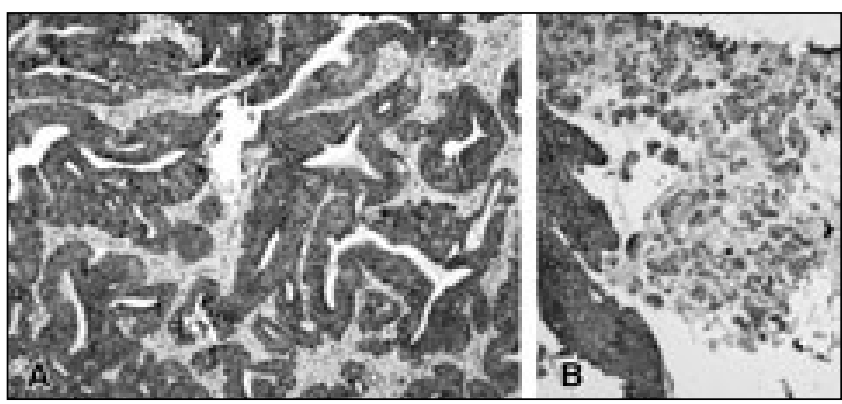

Figure 4) Immunostaining for cytokeratin (AE1/AE3) shows strong positivity in tumour cells of the skin (A) (original magnification $\times 400$ ); and esophagus $(\mathbf{B})$ (original magnification $\times 100$ )

work-up at that time showed no evidence of extracutaneous metastasis. The patient was unique in that she had skin metastasis for one month as the initial manifestation of a primary esophageal adenocarcinoma in the absence of any local symptoms. The patient was given chemotherapy. After six months of chemotherapy there was no change in the size of the tumour and rebiopsy revealed the same histopathology as the initial specimen.

\section{Histopathological features}

Biopsy of the facial skin revealed unremarkable epidermis and involvement of the dermis by an epithelial tumour showing glandular structure with moderate cellular pleomorphism (Figure 1). Biopsy of the esophageal mucosa showed a similar epithelial-type tumour, with occasional glandular structures and composed of cells with eccentric, hyperchromatic nuclei (Figure 3).

\section{Immunohistochemical findings}

For the immunohistochemical studies, formalin-fixed and paraffin-embedded sections were stained using the standard avidin-biotin-peroxidase complex method. The following monoclonal antibodies were applied: AE1/AE3, 1:50 dilution, Dako A/S, Denmark; CK7 (QV-TL12/30), 1:50 dilution, Dako; CK20, 1:100 dilution, Dako; epithelial membrane antigen, 1:200 dilution, Dako; p53 (DO7), 1:100 dilution, Dako; and c-erbB-2, 1:80 dilution, (Dako). 

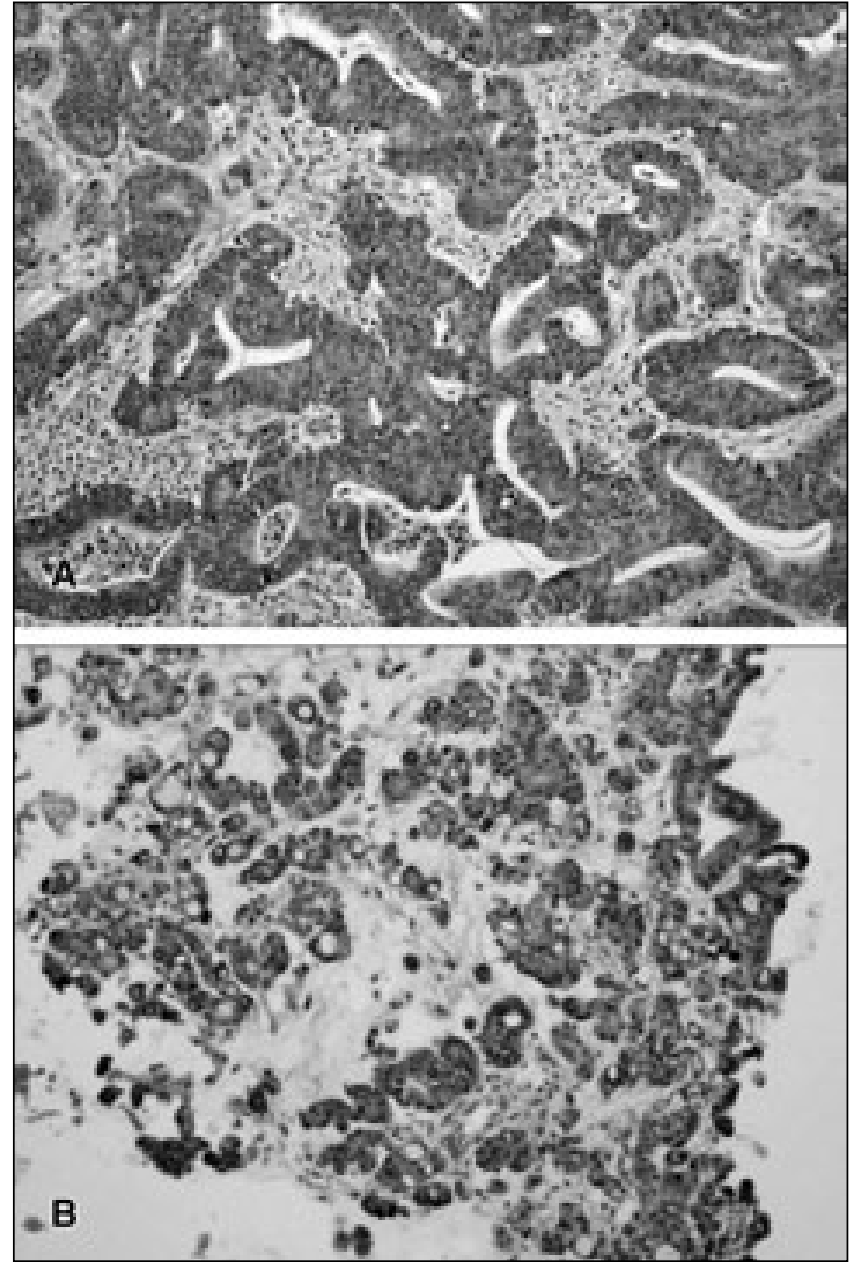

Figure 5) Immunohistochemical staining for CK7 shows positivity for both the skin metastasis (A) (original magnification $\times 400$ ); and esophageal tumour cells $($ B) (original magnification $\times 100$ )

Diaminobenzidine was used to visualize the reaction product. CK7 and CK20 were treated with citrate and microwaved for antigen retrieval.

The immunohistochemical features in the metastatic skin tumour were similar to those in the esophageal adenocarcinoma. The immunohistochemical study indicated an epithelial origin for both the metastatic and the primary tumour.

Both the skin and the esophageal tumour cells showed diffuse immunostaining with AE1/AE3 (Figures 4A and 4B) and diffuse membrane staining with epithelial membrane antigen. Immunostaining was negative for CK20, but positive for CK7 in both samples (Figures 5A and 5B). p53 demonstrated diffuse nuclear staining in both specimens (Figures 6A and 6B). No immunostaining was noted for c-erbB-2 in the tumour cells of skin and of the esophagus.

The immunohistochemical staining (Table 1) provided evidence that the skin metastasis originated from the esophageal carcinoma.

\section{DISCUSSION}

Cutaneous metastases are the 12th most frequent site for all tumour types (7). Skin infiltration may be the first sign of carcinoma, or it may accompany other symptoms or be noted

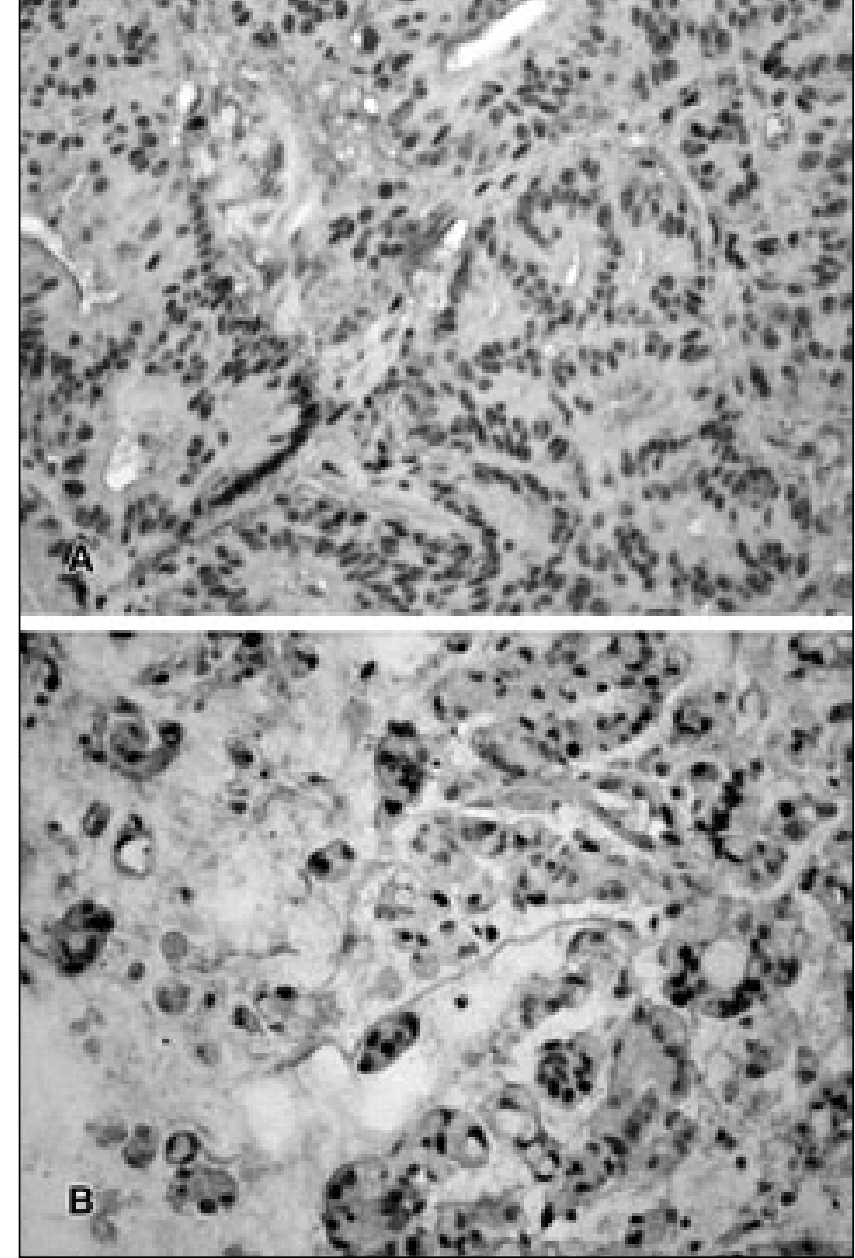

Figure 6) Immunohistochemical staining for $p 53$ is positive in the skin (A) and in the esophageal tumour cells (B). Original magnification $\times 200$

TABLE 1

The result of immunohistochemical staining of the skin metastasis and the esophageal carcinoma

\begin{tabular}{lcccccc}
\hline \multicolumn{2}{r}{ CK (AE1/AE3) } & EMA & CK20 & CK7 & p53 & c-erbB-2 \\
\hline Primary tumour & + & + & - & + & + & - \\
Secondary tumour & + & + & - & + & + & - \\
\hline
\end{tabular}

EMA Epithelial membrane antigen

during follow-up (4).The incidence of skin metastasis is $1 \%$ to $2.7 \%(1)$. The scalp is a relatively common site of cutaneous metastasis (8). Brownstein and Helwig (2) reported that the scalp was the site in $4 \%$ of all skin metastases. Metastases to the skin from various internal organs are uncommon (7). It has been claimed that skin metastases tend to be close to the site of the primary tumour: chest in breast and lung cancers, abdominal wall in gastrointestinal tumours and lower back in renal cell carcinomas (9). Breast cancer is the most common origin of cutaneous metastasis in women, while lung cancer is the most common origin in men (9). Frequency of skin metastases from cancer of internal organs ranges from $0.7 \%$ to $9 \%$ (4). Skin metastases from primary gastric carcinoma are very uncommon (10); the incidence varies from $0.04 \%$ in clinical series to $9 \%$ in 
autopsies (7). Skin metastases are classified macroscopically as nodular, inflammatory or sclerodermoid (1). They have been reported occasionally as multiple ulcerated and erysipeloid lesions (10), and as large nodular lesions involving parts of the neck and trunk (11). In some cases, skin metastases are multiple, firm, nonulcerated nodules (1). It should be noted that solitary lesions may be misdiagnosed as primary skin tumours (1).

Nodular lesions tend to appear suddenly (1). Inflammatory changes indicate rapid spread and deposition of cancer in the subepidermal lymph vessels (1). In the so-called carcinoma erysipeloides, tumour cells fill the lumina of dermal vessels (12). Cutaneous metastases of alimentary tract occur most commonly on the abdominal wall and can be the initial manifestation of a primary gastric tumour (5). Gastric adenocarcinomas spread chiefly by direct invasion and extension to regional lymph nodes and the liver (11). They may occasionally spread via lymphatic and blood vessels. Skin metastases develop most often after invasive procedures for diagnostic or therapeutic purposes as local skin metastasis or seedings (7), mainly in the epigastric and mammary regions (12). Because carcinomas spread preferentially by lymphatic vessels, gastrointestinal tumours may give rise to metastasis in the cervical lymph nodes. It was suggested that an aggressive clone of gastric adenocarcinoma cells metastasizes to cervical lymph nodes and subsequently invades the skin, imitating a primary skin lesion (11). It was also claimed that a clonal population of carcinoma cells exists, with a high affinity for the skin and a low affinity for other organs (5).

Cutaneous metastases are most often rapidly progressing late events with a poor prognosis (7). Our patient, however, had metastatic carcinoma of the skin manifesting as a clinically benign, small, solitary nodule with no other symptoms. The follow-up computed tomography scan revealed thickening of

\section{REFERENCES}

1. Charalambous C, Zipitis CS, Midwinter M. Gastric adenocarcinoma metastatic to the skin: A report. Eur J Cancer Care (Engl) 2002;11:143-4.

2. Brownstein MH, Helwig EB. Metastatic tumors of the skin. Cancer 1972;29:1298-307.

3. Lookingbill DP, Spangler N, Sexton FM. Skin involvement as the presenting sign of internal carcinoma. A retrospective study of 7316 cancer patients. J Am Acad Dermatol 1990;22:19-26.

4. Ozakyol AH, Saricam T, Pasaoglu O. A rare entity: Cutaneous metastasis from gastric adenocarcinoma. Am J Gastroenterol 1999;94:1118-9.

5. Sood A, Midha V, Sekhon JS, Sidhu SS. Generalized cutaneous metastases from carcinoma stomach. Am J Gastroenterol 1998;93:1601.

6. Smith KJ, Williams J, Skelton H. Metastatic adenocarcinoma of the esophagus to the skin: New patterns of tumor recurrence and alternate treatments for palliation. J Cutan Pathol 2001;28:425-31.

7. Garcia-Gonzalez E, Alvarez-Paque L, Loyola-Zarate M, the gastric wall in the cardia, and the patient had no epigastric pain, epigastric discomfort, dyspepsia or regurgitation, and no weight loss. A barium swallow revealed esophageal mucosal irregularities, despite an absence of reflux or difficulties in swallowing. Esophageal biopsy revealed an immunohistochemically CK7+/CK20 - invasive adenocarcinoma. To determine the primary site, immunostaining for keratins can provide important information (9). Although exceptions exist, $\mathrm{CK} 7+/ \mathrm{CK} 20+$ is apparent in one-third of gastric adenocarcinomas and CK7-/CK20+ in another one-third of gastric adenocarcinomas (9), while $\mathrm{CK} 7+/ \mathrm{CK} 20-$ is reported in esophageal adenocarcinomas (6).

Squamous cell carcinomas accounted for $90 \%$ of esophageal cancers before 1970; currently, adenocarcinomas are assumed to be increased in number (6). It was concluded that nearly all cases of esophageal adenocarcinomas arise from a premalignant lesion of esophagus, known as Barrett's esophagus $(6,12)$. In our patient, however, no features of Barrett's esophagus were detected in the esophageal sample. Although Barrett's esophagus is recognized as a precursor lesion, its etiology and prevalence remain unclear (13). Strong association among Barrett's esophagus, esophageal adenocarcinoma and gastroesophageal reflux has been reported (13). Our patient was, however, symptomless.

Our case emphasizes that, in staging of esophagogastric carcinoma, skin lesions should be looked for and biopsied because the findings can determine further management. Newly appearing skin lesions may be the first presentation of an advanced visceral cancer and should be appropriately explored (1). Although cancers of internal organs rarely present with skin metastases, nonhealing dermal ulcers, persistent indurated erythema and skin nodules of undetermined cause need to be biopsied so as not to miss cutaneous metastases of an unknown primary cancer (4).

Lisker-Melman M. Seeding of gastric adenocarcinoma cells to the skin after invasive procedures. J Clin Gastroenterol 1998;26:82-4.

8. Kim HJ, Min HG, Lee ES. Alopecia neoplastica in a patient with gastric carcinoma. Br J Dermatol 1999;141:1122-4.

9. Rosai J. Rosai and Ackerman's Surgical Pathology, 9th edn. London: CV Mosby Publishing Company, 2004:55,203.

10. Ikeda Y, Niimi M, Takami H, Kodaira S. Successfully treated carcinoma erysipeloides from gastric cancer. Ann Oncol 2003;14:1328-9.

11. Essa K, Pervez S, Shah LM, Soomro IN. Signet cell gastric carcinoma presenting as multiple large skin nodules. Australas J Dermatol 2001;42:219-20.

12. Hamamoto Y, Nagai K, Ichimiya M, Yamamoto K, Kinoshita E, Muto M. Regressive effect of intralesional injection of a moderate dose of recombinant interleukin-2 on carcinoma erysipeloides from gastric carcinoma. Clin Exp Dermatol 2001;26:42-4.

13. Kim R, Weissfeld JL, Reynolds JC, Kuller LH. Etiology of Barrett's metaplasia and esophageal adenocarcinoma. Cancer Epidemiol Biomarkers Prev 1997;6:369-77. 


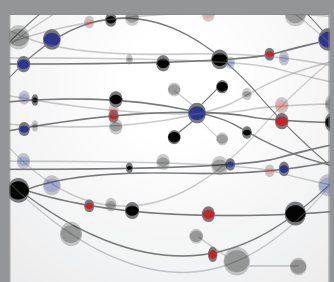

The Scientific World Journal
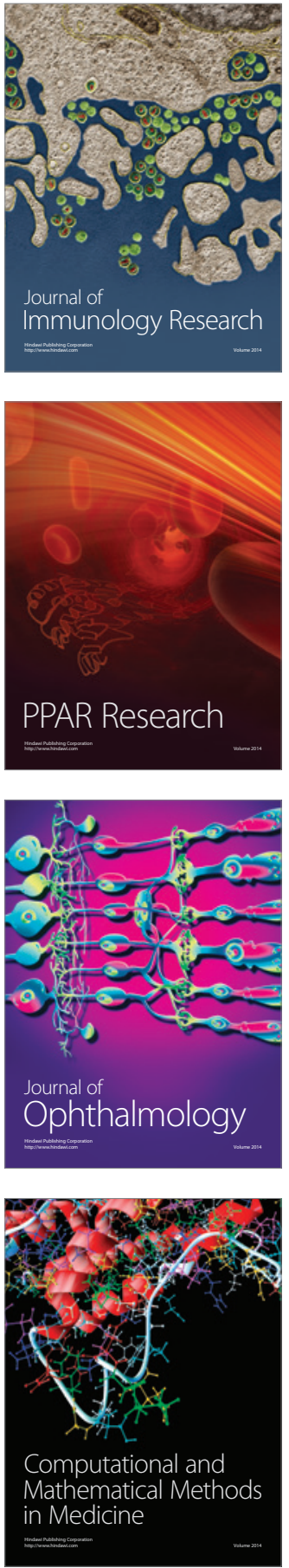

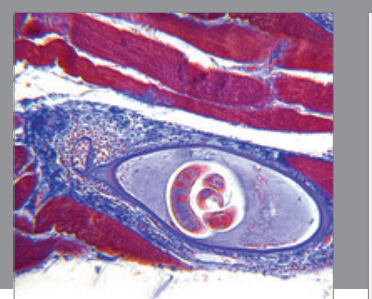

Gastroenterology Research and Practice

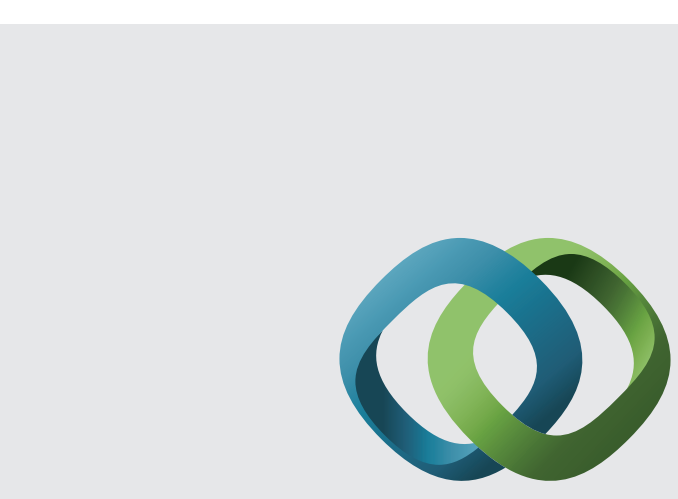

\section{Hindawi}

Submit your manuscripts at

http://www.hindawi.com
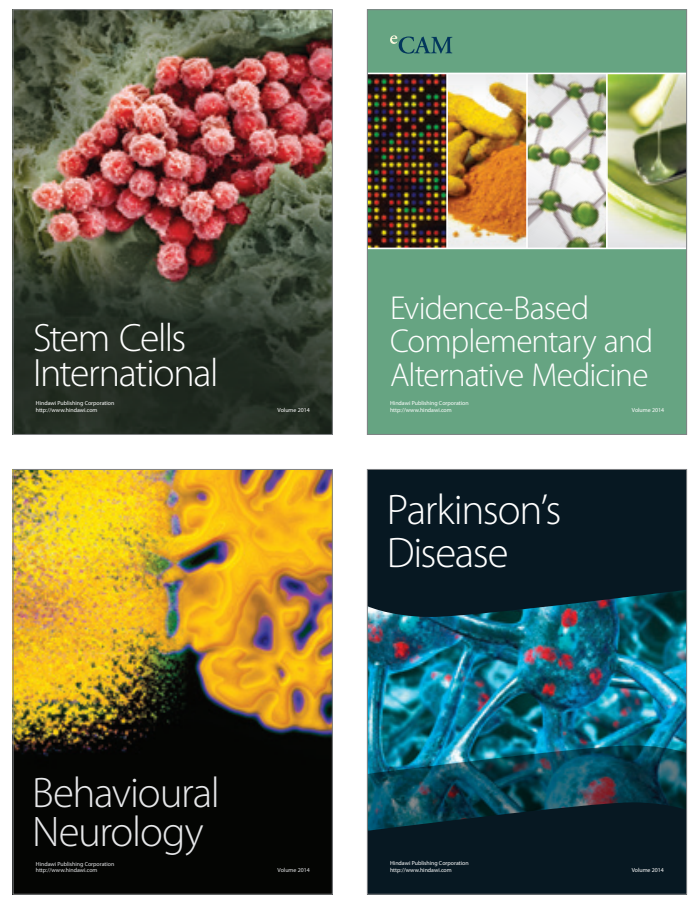
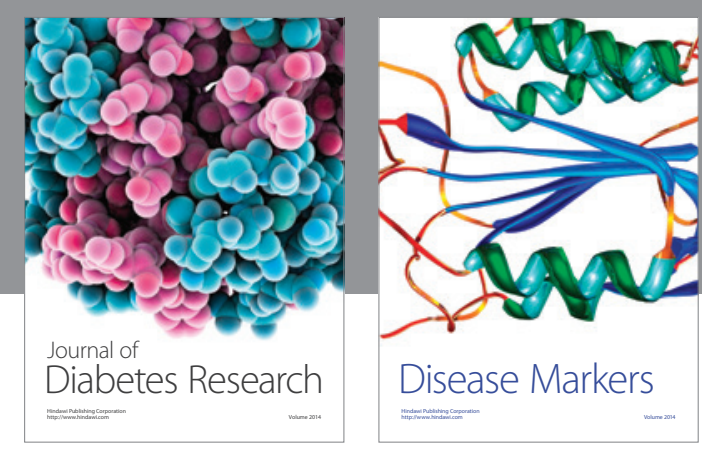

Disease Markers
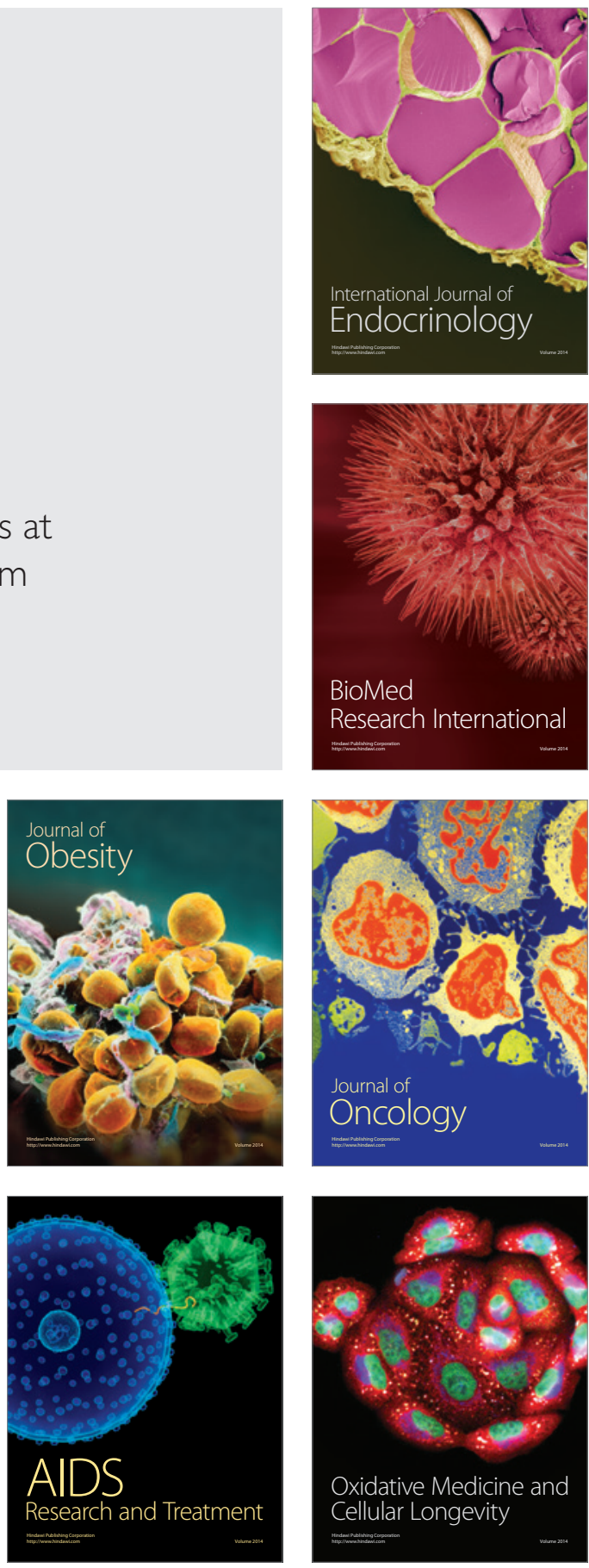